DOI: $10.1007 / \mathrm{s} 00350-014-3644-0$

\section{Entfall des Honoraranspruchs bei Teilbarkeit ärztlicher Leistungen}

BGB $\S 280$ Abs. 1, 611, 628 Abs. 1, 812 Abs. 1; ZPO §114

Ein Honoraranspruch kann unbegründet sein, soweit er auf Bezahlung einer für den Beklagten infolge eines Behandlungsfehlers unbrauchbaren Leistung, nämlich die Oberkieferbrücke gerichtet ist. Das darauf gestützte Teilhonorar ist aus dem Gesamtanspruch herauszurechnen (vgl. auch Senat, Beschl. v. 1.7.2010 - $20 \mathrm{~W}$ 23/10 -).

KG, Beschl. v. 14.1.2013 - $20 \mathrm{~W} 83 / 12$ (LG Berlin)

Problemstellung: In der Rechtsprechung der Obergerichte ist anerkannt, dass der Honoraranspruch eines Zahnarztes unter bestimmten Voraussetzungen entfallen kann. Dabei wird entweder auf die vollständige Unbrauchbarkeit der zahnärztlichen Leistung (vgl. OLG Düsseldorf, VersR 1985, 456; OLG Koblenz, MedR 2010, 263) oder auf die Schwere der Pflichtverletzung (vgl. OLG Nürnberg, GesR 2008, 363) abgestellt. Im ersten Fall verlangt die Rechtsprechung, dass etwa bei zahnprothetischen Anfertigungen und Einpassungen eine Nachbesserung ausscheiden und nur eine Neuanfertigung in Betracht kommen dürfe. Im zweiten Fall wird ein besonders grobes oder vorsätzliches Fehlverhalten verlangt, so dass sich die Honorarforderung als missbräuchliche Rechtsausübung darstellte. Beiden Ansätzen ist jedoch die Aussage gemein, dass ärztliche Schlechtleistungen nur die Wirkung eines vollständigen Entfalls der Honorarforderung zur Folge haben können, eine Minderung jedoch mangels vorgesehener gesetzlicher Regelung im Dienstvertragsrecht nicht in Betracht komme.

Das KG schließt sich in der vorliegenden Entscheidung der ersten Begründungslinie an, splittet sodann aber die erbrachten zahnärztlichen Leistungen nach den einzelnen prothetischen Anfertigungen und Einpassungen auf. Faktisch deutet das KG hierdurch die Möglichkeit einer bislang nicht akzeptierten Honorarminderung an, indem es zulässt, dass einzelne Elemente der Behandlung als vollständig unbrauchbar zu qualifizieren sein können. Eine ärztliche Behandlung kann je nach Präzision der Argumentation jedoch häufig in Parzellen zerlegt werden. Der Verweis auf den Senatsbeschluss des KG vom 1.7.2010 - 20 W 23/10 -, MedR 2011, 45, ist ebenfalls nicht weiterführend, da im dort entschiedenen Fall die gesamte Prothetik als unbrauchbar qualifiziert wurde, die Behandlung somit nicht in brauchbare und unbrauchbare Einzelteile zu zerlegen war.

Die Entscheidung des KG zeigt einmal mehr die Unausgewogenheit der Rechtsprechung im Umgang mit dem ärztlichen Honoraranspruch. Einerseits soll der ärztliche Dienstleister gegen Minderungen geschützt werden und nur für kausal verursachte Folgeschäden haften müssen. Auf der anderen Seite soll diesem Schutz eine äußere Grenze gesetzt sein, die in der Unbrauchbarkeit der Leistung gesehen wird. Allerdings ist nach wie vor nicht ersichtlich, welche ärztliche Leistung im Einzelnen die genaue Beurteilungsgrundlage bildet. Zudem ist diese Rechtsprechung vor dem Hintergrund des Gleichheitssatzes kritisch zu betrachten, da Arzte, die weitestgehend nicht aufspaltbare Leistungen erbringen, im Falle von Behandlungsfehlern einen erhöhten Schutz ihres Honorars genießen. Das überzeugt nicht.

Bearbeitet von Wiss. Ass. Dr. iur. Jens Prütting, LL. M. oec., Universität Freiburg i. Br., Institut für Wirtschaftsrecht, Abt. 1, Wilhelmstr. 26, 79098 Freiburg i.Br., Deutschland
Zum Sachverhalt: Die K1. war bei der Bekl. in zahnärztlicher Behandlung. Sie erhielt zunächst eine vorläufig eingegliederte implantatgestützte Prothese und einige Tage später eine endgültige Eingliederung des Zahnersatzes. Sodann brach die Kl. die weitere medizinische Versorgung bei der Bekl. ab und zahlte das bis dahin angefallene Honorar. Nunmehr begehrt sie Rückzahlung wegen erheblicher Mängel und behaupteter Disfunktionalität des Zahnersatzes.

Aus den Gründen: Die Beschwerde mußte teilweise Erfolg haben. Die Rechtsverteidigung des Bekl. hat hinreichende Aussicht auf Erfolg, soweit er sich gegen die auf Herstellung der Oberkieferbrücke gestützte, an die Kl. abgetretene Honorarforderung des Zahnarztes wendet. Die Sachverständige hat im selbständigen Beweisverfahren $35 \mathrm{OH}$ 5/11 durch Gutachten v. 31.10.2011 festgestellt, daß die Oberkieferbrücken nach einer angemessenen Wartezeit nach erneuter Wurzelkanalbehandlung zu erneuern sind, und daß dies auch für die Krone auf Zahn 46 gilt [...]. Mithin kann unter weiterer Berücksichtigung der Ausführungen der Sachverständigen zu den Fehlerursachen [...] nicht ausgeschlossen werden, $\mathrm{da}$ die Oberkieferbrücken behandlungsfehlerhaft hergestellt wurden. Hierüber hat das LG ggf. Beweis zu erheben.

Danach wäre der Honoraranspruch unbegründet, soweit er auf Bezahlung einer für den Bekl. infolge eines Behandlungsfehlers unbrauchbare[n] Leistung, nämlich die Oberkieferbrücke gerichtet ist. Das darauf gestützte Teilhonorar ist aus dem Gesamtanspruch herauszurechnen (vgl. auch Senat, Beschl. v. 1.7.2010 - 20 W 23/10 -).

Der teilweisen Bewilligung von Prozeßkostenhilfe steht nicht entgegen, daß die Rechtsverteidigung des Bekl. nur gegenüber einem Teilanspruch der Kl. erfolgreich erscheint, und daß sich dieser Teil zur Zeit betragsmäßig nicht hinreichend darstellt. Zwar darf Prozeßkostenhilfe nicht für unselbständige Verfahrensabschnitte bewilligt werden, wie etwa eine von mehreren Einwendungen (Geimer, in: Zöller, ZPO, 29. Aufl. 2012, \$114, Rdnr. 20). Dies kann jedoch nicht gelten, soweit sich die Verteidigung, wie hier, gegen sachlich und betragsmäßig abgrenzbare Teile eines Gesamtanspruchs richtet, für die auch dem Anspruchsteller unter den geforderten Voraussetzungen Prozeßkostenhilfe zu bewilligen wäre.

Soweit die Beschwerde zurückzuweisen war, folgt der Senat den zutreffenden Gründen der angefochtenen Entscheidung in Verbindung mit dem Nichtabhilfebeschluß der Kammer v. 5.12.2012

Prozeßkostenhilfe für dieses Beschwerdeverfahren konnte nicht bewilligt werden (vgl. Geimer, a.a. O., $\$ 114$, Rdnr. 3 a.A.).

$[\ldots]$

\section{Keine Haftung für Fehleinschätzung des konsul- tierten Facharztes; Zulässigkeit einer erst in zwei- ter Instanz auf den Facharzt erweiterten Klage}

BGB $\S \S 249,253,276,278,280,611,823,831$; ZPO §§263, $264,525,533$

1. Grundsätzlich darf ein Hausarzt der ihm mitgeteilten Einschätzung eines Facharztes vertrauen, es sei denn, dessen Sicht begegnet nach den sonstigen Erkenntnismöglichkeiten des Hausarztes erheblichen Zweifeln, weil sie nicht mit dessen eigenen Befunden oder sonstigen medizinischen Wahrnehmungen zu vereinbaren sind und daher Rückfragen bei dem Facharzt oder Erörterungen mit dem Patient gebieten.

Eingesandt von RiOLG Ernst Weller, Koblenz, Deutschland; bearbeitet von Wiss. Mitarb. Charlotte Landwehr, Universität zu Köln, Institut für Medizinrecht, Albertus-Magnus-Platz, 50923 Köln, Deutschland 